\title{
Attenuation of Endotoxin-Induced Acute Lung Injury by the Rho-Associated Kinase Inhibitor, Y-27632
}

\author{
Sadatomo Tasaka, Hidefumi Koh, Wakako Yamada, Mie Shimizu, Yuko Ogawa, Naoki Hasegawa, \\ Kazuhiro Yamaguchi, Yoshiki Ishii, Sarah E. Richer, Claire M. Doerschuk, and Akitoshi Ishizaka
}

Department of Medicine, Keio University School of Medicine, Tokyo; Department of Pulmonary Medicine and Clinical Immunology, Dokkyo University School of Medicine, Tochigi, Japan; and Department of Pediatrics, Case Western Reserve University, Cleveland, Ohio

\begin{abstract}
A small GTPase, Rho, plays key roles in cell adhesion, motility, and contraction after stimulation. Among Rho effectors isolated, the family of Rho-associated coiled-coil-forming protein kinases (ROCK) is implicated in Rho-mediated cell adhesion and smooth muscle contraction. The effect of a specific inhibitor of ROCK, Y-27632, was evaluated in a murine model of acute lung injury induced by intravenous injection of Escherichia coli endotoxin (lipopolysaccharide [LPS]). Lung edema was evaluated by measuring extravascular leakage of radio-labeled serum albumin, and neutrophil emigration into the lung parenchyma by morphometric observation and measuring myeloperoxidase activity. Pretreatment with Y-27632 attenuated both lung edema and neutrophil emigration after LPS. We also measured albumin transfer through cultured endothelial cell monolayers on a porous filter. Tumor necrosis factor- $\alpha$ significantly increased albumin transfer, which was attenuated by pretreatment with Y-27632. Fluorescence microscopy revealed that morphologic changes in endothelial cells induced by tumor necrosis factor- $\alpha$ were inhibited by Y-27632. In contrast, the increased fraction of neutrophils with polymerized actin after formyl-methionyl-leucyl-phenylalanine was not altered by Y-27632. These data suggest that ROCK may play an important role in the pathogenesis of LPS-induced lung injury and that ROCK inhibition could attenuate cytoskeletal rearrangement of endothelial cells, leading to decreased neutrophil emigration into the lung parenchyma.
\end{abstract}

Keywords: lipopolysaccharide; lung; neutrophils; rodent

Acute respiratory distress syndrome (ARDS) is a critical illness, which is characterized by acute lung injury leading to permeability pulmonary edema and respiratory failure $(1,2)$. Circulating neutrophils play a major role in the development of acute lung injury in both clinical settings and experimental animal models $(3,4)$. Neutrophil sequestration into the pulmonary microvasculature and migration into the lung parenchyma are critical steps in the pathogenesis of acute lung injury, which is thought to be induced by inflammatory mediators binding to specific receptors on neutrophils and endothelial cells $(4,5)$. Previous studies suggest that the sequestration of neutrophils occurs through at least two sequential events $(4,6,7)$. The first event is a rapid stiffening of neutrophils, which occurs during the first few minutes after an inflammatory mediator enters the bloodstream. Neutrophil stiffening depends upon the polymerization of globular $(\mathrm{g})$ actin to filamentous (f) actin to form an actin-rich rim beneath the plasma membrane $(5,8)$. The second event is firm adhesion mediated by CD11/CD18 and other adhesion molecules, which is necessary to maintain the sequestered neutrophils within the capillaries $(3,4)$. This firm adhesion is followed by transendothe-

(Received in original form January 9, 2004 and in final form January 25, 2005)

Correspondence and requests for reprints should be addressed to Akitoshi Ishizaka, M.D., Ph.D., Department of Medicine, Keio University School of Medicine, 35 Shinanomachi, Shinjuku-ku, Tokyo 160-8582, Japan. E-mail: ishizaka@cpnet.med. keio.ac.jp

Am J Respir Cell Mol Biol Vol 32. pp 504-510, 2005

Originally Published in Press as DOI: 10.1165/rcmb.2004-00090C on March 18, 2005

Internet address: www.atsjournals.org lial migration into the lung parenchyma, leading to neutrophilmediated tissue injury.

Rho is a monomeric, 21-kD GTPase that exists in at least three isoforms in mammals (Rho A, Rho B, and Rho C), which increases cell adhesion, formation of actin stress fibers, and cell contraction after thrombin stimulation (9-11). In cells other than leukocytes, Rho activity is required for the formation of actin stress fibers (10). In human neutrophils, Rho plays an important role in signaling for myosin light chain phosphorylation and cell migration (12). Among Rho effectors isolated, the family of Rho-associated coiled-coil-forming protein kinases (ROCK) is implicated in Rho-mediated cell adhesion and smooth muscle contraction $(13,14)$.

Y-27632 is a selective ROCK inhibitor, which has been shown to act as a bronchodilator and vasodilator through relaxation of smooth muscle (15-18). To determine the role of Rho/ROCK in the pathogenesis of acute lung injury, we investigated the effect of Y-27632 on lipopolysaccharide (LPS)-induced acute lung injury using a murine model. Lung edema was evaluated by measuring extravascular leakage of radio-labeled albumin, and neutrophil emigration into the lung parenchyma by morphometric observation. We also measured albumin transfer through cultured endothelial cell monolayers on a porous filter and evaluated the effect of Y-27632 on the changes in albumin transfer after TNF- $\alpha$ stimulation. Confocal microscopy was performed to examine the effect of Y-27632 treatment on morphologic changes in endothelial cells and neutrophils induced by tumor necrosis factor (TNF)- $\alpha$ and fMLP, respectively.

\section{MATERIALS AND METHODS}

\section{Animals and Antibodies}

C57BL/6 mice, 8-11 wk old, were purchased from CLEA Japan (Tokyo, Japan). All mice were housed in the Keio University Animal Resource Center, and all experiments received institutional approval, conforming to NIH guidelines. A ROCK inhibitor, Y-27632, was kindly provided by Mitsubishi Pharma Corporation (Osaka, Japan). Escherichia coli endotoxin (serotype B:55) and fMLP were purchased from Sigma Chemicals (St. Louis, MO). Recombinant human TNF- $\alpha$ was obtained from PeproTech, Inc. (Rocky Hill, NJ).

\section{Protocol 1: Effect of Y-27632 on LPS-Induced Acute Lung Injury}

Thirty-two C57BL/6 mice were divided into four groups: Group 1 was given an intraperitoneal injection of $200 \mu \mathrm{l}$ of phosphate-buffered saline (PBS) before intravenous injection of PBS $(50 \mu \mathrm{l})$; Group 2 received

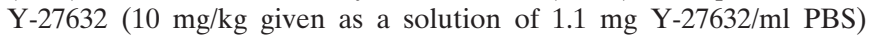
before intravenous PBS; Group 3 received intraperitoneal injection of PBS followed by intravenous injection of E. coli LPS (3 mg/kg given as a solution of $1.2 \mathrm{mg}$ LPS/ml PBS); Group 4 was treated with Y-27632 before LPS challenge. Mice were anesthetized using ketamine hydrochloride ( $80-100 \mathrm{mg} / \mathrm{kg}$ intramuscularly) and acepromazine maleate $(5-10 \mathrm{mg} / \mathrm{kg}$ intramuscularly) and received intravenous injection of ${ }^{125} \mathrm{I}$-labeled bovine albumin $(0.1 \mathrm{mCi} / \mathrm{mouse})$ to measure edema. Either PBS or LPS was injected intravenously $20 \mathrm{~min}$ after intraperitoneal injection of PBS or Y-27632. Blood samples were collected by orbital 
sinus puncture immediately before and $15 \mathrm{~min}$ after intravenous injection. Two minutes before the end of the study, ${ }^{131}$ I-labeled human albumin $(0.1 \mathrm{mCi} / \mathrm{mouse})$ was injected to measure the intravascular blood volume. After $6 \mathrm{~h}$, the lungs were removed and fixed using intratracheal instillation of $6 \%$ glutaraldehyde at $22 \mathrm{~cm} \mathrm{H}_{2} \mathrm{O}$. Blood samples were obtained from the orbital sinus (time 0 and $15 \mathrm{~min}$ ) and inferior vena cava $(6 \mathrm{~h})$. White blood cells (WBC) were counted using a hemocytometer, and differentials were determined using blood smears stained with Diff-Quik (American Scientific Products, McGraw, IL).

Edema formation in lungs was evaluated by quantitating the accumulation of extravascular albumin (EVA) as previously described $(7,19)$. The isotope-specific radioactivity of excised, fixed lungs as well as samples of blood and plasma was measured (ARC-300; Aloka, Tokyo, Japan) before sectioning the lungs for morphometric analysis. Blood and plasma volumes of lungs were determined from ${ }^{125} \mathrm{I}$-albumin and ${ }^{131} \mathrm{I}$-albumin counts, respectively. Pulmonary edema (EVA in lungs) was calculated as total lung albumin content minus intravascular lung albumin content. Intravascular lung albumin content was calculated from the pulmonary blood volume and the hematocrit for each animal.

Pulmonary neutrophils were quantified by morphometric analysis in histologic sections (20). Paraffin-embedded histologic 5- $\mu \mathrm{m}$ sections of lungs were cut and stained with hematoxylin and eosin. Neutrophil emigration was quantitated by counting the number of neutrophils in 200 randomly selected alveoli and was expressed as the number of neutrophils per 100 alveoli.

In separate experiments, the neutrophil accumulation within the lungs was evaluated by measuring the myeloperoxidase (MPO) activity of lung tissue samples, following a previously reported method (21). Twenty-four C57BL/6 mice were divided into four groups as described above and killed $6 \mathrm{~h}$ after intravenous injection of either PBS or LPS. After lungs were perfused via the right ventricle with $3 \mathrm{ml}$ of sterile PBS, the lungs were snap-frozen in liquid nitrogen and stored at $-70^{\circ} \mathrm{C}$. After weighing, whole lungs were homogenized in $0.5 \%$ hexadecyltrimethylammonium bromide in $50 \mathrm{mM}$ potassium phosphate buffer $(\mathrm{pH} \mathrm{6.0)}$ in an ice bath. The specimens were freeze-thawed three times, after which sonication was repeated. Suspensions were then centrifuged at 40,000 $\times g$ for $30 \mathrm{~min}$ at $4{ }^{\circ} \mathrm{C}$. Then $0.1 \mathrm{ml}$ of supernatant was combined with $2.9 \mathrm{ml}$ of $50 \mathrm{mM}$ potassium phosphate buffer $(\mathrm{pH} \mathrm{6.0)}$ containing $0.167 \mathrm{mg} / \mathrm{ml}$ of $o$-dianisidine dihydrochloride and $0.0005 \%$ hydrogen peroxide. The enzymatic activity was determined spectrophotometrically as the change in absorbance at $460 \mathrm{~nm}$ over 5 min using a 96-well plate reader (Molecular Devices, Sunnyvale, CA). MPO activity is expressed per gram of lung weight.

\section{Protocol 2: Effect of Y-27632 on Neutrophil Cytoskeleton}

Human neutrophils were isolated from peripheral blood of healthy volunteers using a two-step density gradient of Histopaque 1077 and 1119 (Sigma Chemicals) as previously described (22). The neutrophil purity was $>95 \%$. The isolated neutrophils were fixed in the presence of $10 \%$ paraformaldehyde for 2 min after treatment with fMLP or PBS. Neutrophils were then permeabilized with $0.2 \mathrm{mg} / \mathrm{ml}$ of lysophosphatidylcholine, and stained with $0.33 \mu \mathrm{M}$ rhodamine-labeled phalloidin for $60 \mathrm{~min}$ to label filamentous actin (F-actin) (23). After staining, the neutrophils were examined using fluorescence microscopy. Two hundred randomly selected neutrophils were observed, and neutrophil cytoskeletal rearrangement was quantitatively expressed as the percentage of neutrophils containing a submembrane rim of F-actin. Confocal microscopy was performed to compare the structure of the F-actin rim.

\section{Protocol 3: Effect of Y-27632 on Endothelial Cell Damage Induced by TNF- $\alpha$}

Human pulmonary artery endothelial cells (HPAECs) were obtained from Kurabo (Osaka, Japan) at the fourth passage. HPAECs were cultured in a humidified $5 \% \mathrm{CO}_{2}$ atmosphere in the medium provided by the manufacturer (HuMedia; Kurabo) supplemented with $2 \%$ fetal calf serum and used for the experiments between the sixth and seventh passage. Endothelial cell monolayers on filters were prepared as previously described (24). In brief, Millicell-HA tissue culture plate well inserts (Millipore Co., Bedford, MA) were incubated with bovine fibronectin at $37^{\circ} \mathrm{C}$ for $3 \mathrm{~h}$ to facilitate cell attachment. The fibronectin solution was aspirated, and the endothelial cells suspended in the culture medium were seeded on a membrane filter at a density of $4 \times 10^{5}$ cells/ filter insert. The inserts were placed into a 6-well culture plate, where each well was filled with $2 \mathrm{ml}$ of culture medium and incubated at $37^{\circ} \mathrm{C}$ in a humidified $5 \% \mathrm{CO}_{2}$ atmosphere. After 2 wk of culture, HPAECs were found to reach confluence on the filter, and permeability measurements and morphologic analyses were performed.

As an index of permeability, we measured the albumin transferred across a cultured endothelial cell monolayer on a porous filter. HPAECs on the filter were pretreated with $0.1,1,10$, and $100 \mu \mathrm{M}$ of Y-27632 for $30 \mathrm{~min}$ and then incubated with $10^{2} \mathrm{U} / \mathrm{ml}$ of TNF- $\alpha$ for 6 or $24 \mathrm{~h}$. We aspirated the TNF- $\alpha$ solution and added $500 \mu$ l of PBS containing $0.1 \%$ bovine albumin to the upper chamber (i.e., the filter well insert). The insert was then placed in 1 well of a 24-well culture plate, in which each well was filled with $0.7 \mathrm{ml}$ of PBS alone. The solution in the well surrounding the filter insert occupied the lower chamber. The above fluid volumes resulted in equal levels of fluid in the upper and lower chambers and eliminated difference in hydrostatic pressure. After incubation for $20 \mathrm{~min}$, the insert was removed from the well. The albumin concentration of the lower chamber was measured with a Bio-Rad Protein Assay kit (Bio-Rad, Hercules, CA). To evaluate the effect of Y-27632 on unstimulated endothelial cells, albumin transfer was measured across monolayers that were pretreated with $100 \mu \mathrm{M}$ Y-27632 for $30 \mathrm{~min}$ and then incubated with PBS for 6 or $24 \mathrm{~h}$

Morphologic analysis was also performed to assess the changes in the actin microfilament cytoskeleton of endothelial monolayers. Monolayers, which were unstimulated, stimulated with $10^{3} \mathrm{U} / \mathrm{ml}$ of TNF- $\alpha$, or pretreated with Y-27632 $(100 \mu \mathrm{M})$ before TNF- $\alpha$ exposure for $24 \mathrm{~h}$, were stained with rhodamine-phalloidin as previously described (25). After staining, the monolayers were examined and photographed using a fluorescence microscope equipped with epi-illumination (Nikon Optiphot; Nikon, Garden City, NY).

\section{Statistics}

Data are presented as mean \pm SEM. One-way ANOVA and Fisher's least-significant difference test were used. Differences were considered statistically significant when $P<0.05$.

\section{RESULTS}

\section{Pulmonary Edema Formation}

Lung EVA was estimated $6 \mathrm{~h}$ after injection of saline or LPS (Figure 1). LPS induced a significant increase in EVA $(P<$ $0.001)$, and $\mathrm{Y}-27632$ partially attenuated this increase $(P<0.05)$. There was no significant difference in EVA between the two groups that received saline injection with or without Y-27632 pretreatment.

\section{Neutrophil Emigration in Alveolar Spaces}

To evaluate the effect of Y-27632 on LPS-induced neutrophil emigration, emigrated neutrophils were quantified morphologically in histologic sections (Figures $2 \mathrm{~A}$ and $2 \mathrm{~B}$ ). The number of

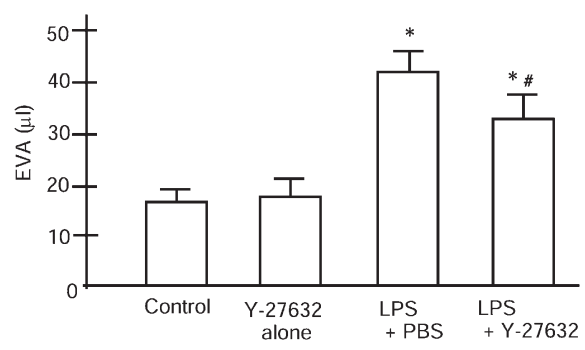

Figure 1. Edema formation $6 \mathrm{~h}$ after intravenous LPS injection. Lung edema was determined by the leakage of radio-labeled albumin. Mice received an intraperitoneal injection of either PBS or Y-27632. Regardless of Y-27632 treatment, mice administered LPS revealed increased EVA $\left({ }^{*} P<0.01\right)$. Pretreatment with $Y-27632$ significantly decreased edema formation after LPS injection ( $P<0.05)(n=8$ in each group). 
neutrophils per 100 cross-sectioned alveolar spaces is shown in Figure 2B. Pretreatment with Y-27632 inhibited neutrophil emigration induced by LPS. Neutrophil emigration was less in mice pretreated with Y-27632 (1,442 \pm 48 neutrophils/100 alveoli) compared with mice pretreated with intraperitoneal PBS $(1,587 \pm 36$ neutrophils/100 alveoli, $P<0.05)$.

MPO activity was measured to evaluate the total neutrophil accumulation within the lungs (Figure 2C). MPO activity in the mice administered LPS without Y-27632 pretreatment was significantly elevated compared with that of the control group $(P<0.01)$. Y-27632 pretreatment significantly attenuated the increase in lung MPO activity induced by LPS $(P<0.05)$. There was no significant difference in MPO activity between the control and LPS + Y-27632 groups.

\section{Peripheral Neutrophil Count}

Peripheral neutrophil counts were determined at time $0,15 \mathrm{~min}$, and $6 \mathrm{~h}$ after injection (Figure 3 ). In mice that received intravenous LPS, the neutrophil count was markedly decreased at $15 \mathrm{~min}$ and had almost recovered by $6 \mathrm{~h}$. At $6 \mathrm{~h}$, the neutrophil count was increased in the control and Y-27632 groups as compared with time 0 . In mice that received LPS, the neutrophil count was less than that in mice without LPS challenge at $6 \mathrm{~h}$. There was no difference in neutrophil counts between mice with and without Y-27632 pretreatment at any time point.

\section{Morphologic Changes in Stimulated Neutrophils}

Neutrophil cytoskeletal rearrangement after fMLP stimulation, which was expressed as the percentage of neutrophils containing an F-actin rim, is shown in Table 1. Among the control neutro-

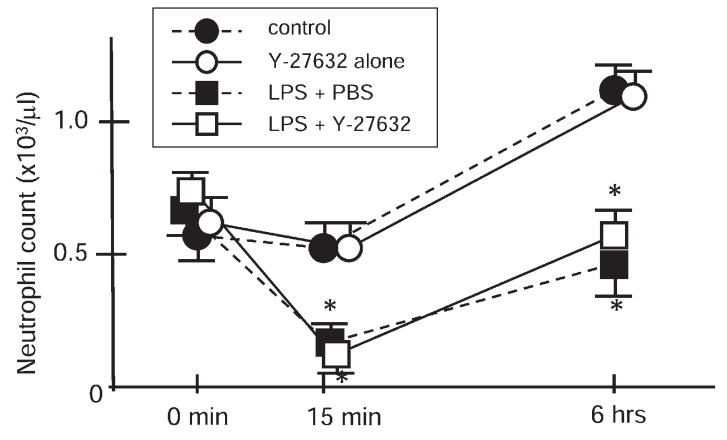

Figure 3. Circulating neutrophil count. Blood samples were obtained from the orbital sinus (time 0 and $15 \mathrm{~min}$ ) and inferior vena cava (6 h). Regardless of Y-27632 treatment, mice administered LPS revealed a significant decline of neutrophil count $\left({ }^{*} P<0.01\right)$. At $6 \mathrm{~h}$, neutrophil count in LPS-treated mice was increased compared with that at 15 min, but was still less than that in mice without LPS administration. Pretreatment with Y-27632 made no difference to the neutrophil count regardless of LPS challenge ( $n=8$ in each group).

phils, $44.3 \pm 3.8 \%$ of neutrophils had F-actin rims beneath the plasma membrane. After fMLP stimulation for $2 \mathrm{~min}$, this percentage increased to $79.3 \pm 11.0 \%$. Pretreatment with Y-27632 caused no significant change in this actin remodeling event induced by fMLP. There was also no significant difference in the appearance of this F-actin rim between the groups without fMLP stimulation with or without Y-27632 pretreatment. Confocal

A
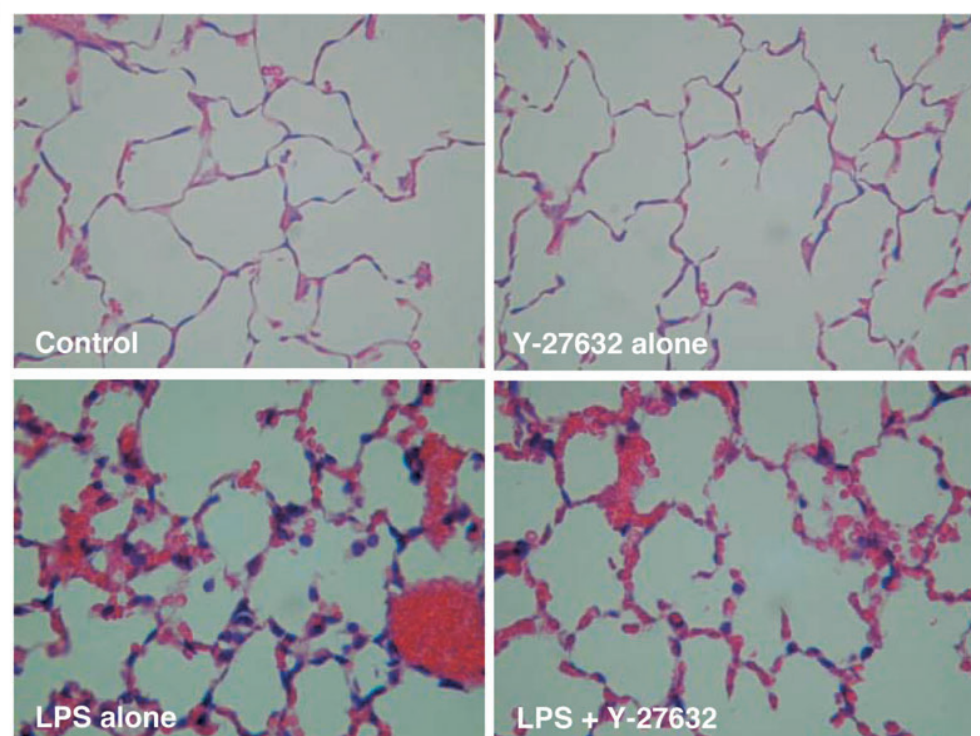

B

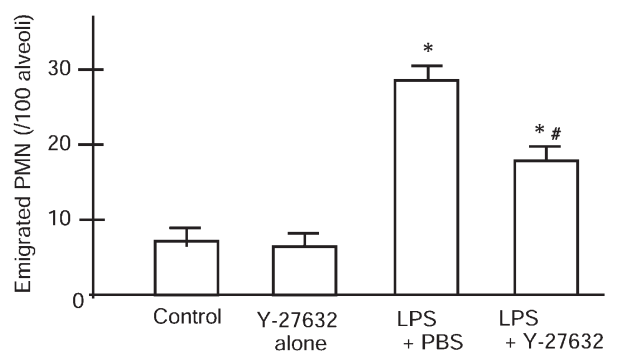

C

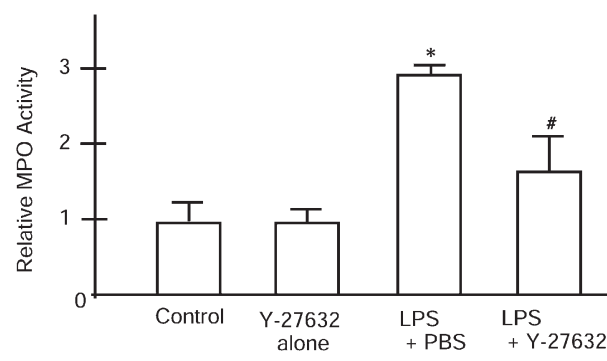

Figure 2. Neutrophil emigration $6 \mathrm{~h}$ after instillation. (A) Representative example of lung pathology. No significant neutrophil emigration was observed in the control mice and the mice with Y-27632 treatment followed by PBS instillation. Marked neutrophil emigration was caused by intratracheal LPS, which was attenuated by the pretreatment with Y-27632. (B) Neutrophil emigration was quantitated by counting the number of neutrophils in 200 randomly selected alveoli and was expressed as the number of neutrophils per 100 alveoli. Regardless of Y-27632 treatment, mice administered LPS revealed increased neutrophil emigration $\left({ }^{*} P<0.01\right)$. Pretreatment with Y-27632 significantly decreased the neutrophil emigration after LPS injection $\left({ }^{\#} P<0.05\right)$ ( $n=8$ in each group). (C) Lung MPO activity. Optical density at $460 \mathrm{~nm}$ of homogenized lung tissue was standardized by lung tissue weight. LPS induced neutrophil emigration $\left({ }^{*} P<0.01\right)$, which was attenuated by $\mathrm{Y}-27632$ pretreatment $\left({ }^{\#} P<0.05\right)$ 
TABLE 1. NEUTROPHILS WITH F-ACTIN RIM APPEARANCE (\%)

\begin{tabular}{lcccc}
\hline & & \multicolumn{3}{c}{$\mathrm{fMLP}(\mathrm{min})$} \\
\cline { 3 - 5 } & PBS & 0.5 & 2 & 5 \\
\hline Control & $44.3 \pm 3.8$ & $78.0 \pm 8.8^{*}$ & $79.3 \pm 11.0^{*}$ & $88.7 \pm 2.8^{*}$ \\
Y-27632 & $38.7 \pm 5.6$ & $81.3 \pm 2.6^{*}$ & $87.3 \pm 1.5^{*}$ & $90.7 \pm 1.3^{*}$
\end{tabular}

Definition of abbreviations: fMLP, formyl-methionyl-leucyl-phenylalanine; PBS, phosphate-buffered saline.

Stimulation with $\mathrm{fMLP}$ rapidly increased neutrophils with an $\mathrm{f}$-actin rim appearance. Treatment with Y-27632 made no difference to the fraction of neutrophils with an f-actin rim. Values are mean $\pm \operatorname{SEM}(n=3)$.

microscopy revealed no differences in the structure of the F-actin rim with or without Y-27632 pretreatment (data not shown).

\section{Albumin Leakage through Endothelial Cell Monolayer}

Changes in the permeability of an endothelial monolayer, which was evaluated after 6 or $24 \mathrm{~h}$ of stimulation by measuring albumin transfer, are shown in Figures $4 \mathrm{~A}$ and $4 \mathrm{~B}$, respectively. Compared with the control, $6 \mathrm{~h}$ stimulation with TNF- $\alpha$ at a concentration of $10^{2} \mathrm{U} / \mathrm{ml}$ caused a significant increase in the albumin concentration in the lower chamber $(P<0.001)$ (Figure 4A). In the groups with Y-27632 pretreatment at concentrations of 1,10 , and $100 \mu \mathrm{M}$, the albumin concentrations of the lower
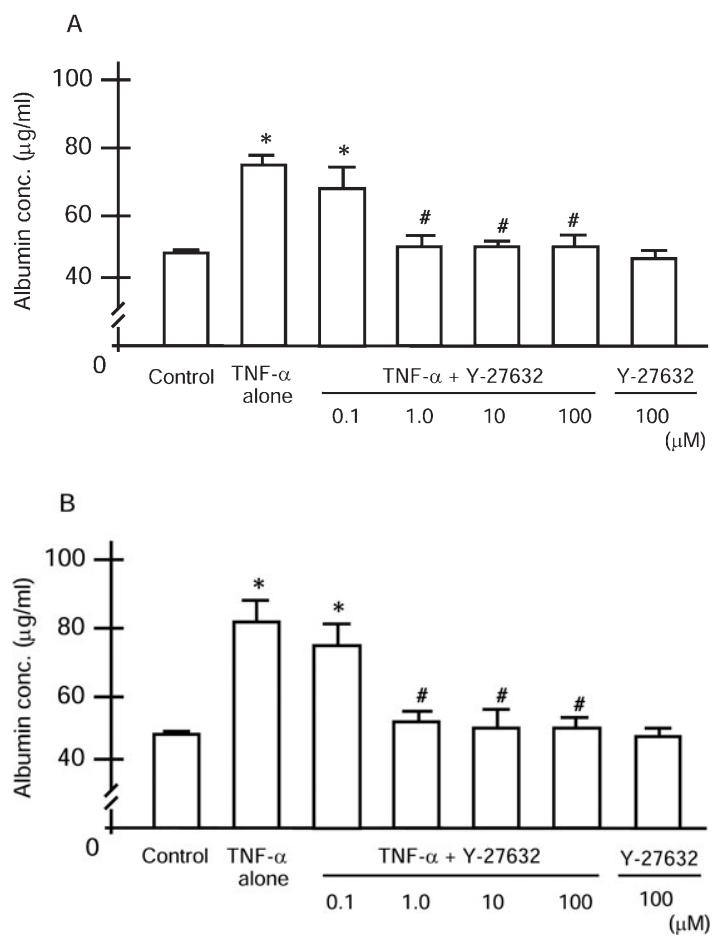

Figure 4. Permeability of endothelial monolayer determined by albumin transfer. TNF- $\alpha\left(10^{2} \mathrm{U} / \mathrm{ml}\right)$ significantly increased the permeability either after 6 or $24 \mathrm{~h}$, compared with the control $\left({ }^{*} P<0.001\right)$. $(A)$ Treatment with 1,10 , and $100 \mu \mathrm{M}$ Y-27632 significantly inhibited the increase in the permeability that was induced by $6 \mathrm{~h}$ stimulation with TNF- $\alpha$ ( $P<0.01$ ), whereas $0.1 \mu \mathrm{M} \mathrm{Y-27632}$ failed to attenuate the damage. $(B)$ The increase in the permeability of endothelial monolayer, which was induced by $24 \mathrm{~h}$ stimulation with TNF- $\alpha$, was completely inhibited by the $\mathrm{Y}-27632(1,10,100 \mu \mathrm{M})$ pretreatment $(\# P<0.01)$. Pretreatment with $0.1 \mu \mathrm{M}$ Y-27632 caused no significant attenuation of the increased permeability of endothelial monolayer. chamber were significantly lower than in the TNF- $\alpha$ control group $(P<0.01)$. There was no significant difference in the albumin concentration of the lower chamber between the TNF- $\alpha$ control group and those with Y-27632 pretreatment at concentration of $0.1 \mu \mathrm{M}$, which indicates that the increase in albumin transfer induced by TNF- $\alpha$ stimulation for $6 \mathrm{~h}$ might be attenuated only by the higher concentration of Y-27632. At 24 h, TNF- $\alpha$ also induced a significant increase in the albumin concentration, compared with the control $(P<0.001)$. Treatment with Y-27632 at concentrations of 1,10 , and $100 \mu \mathrm{M}$ significantly inhibited the increase in albumin transfer induced by $24 \mathrm{~h}$ stimulation with TNF- $\alpha(P<0.01)$, although $0.1 \mu \mathrm{M}$ of $\mathrm{Y}-27632$ made no significant difference in the albumin concentration of the lower chamber (Figure 4B). Y-27632 itself did not induce a change in albumin transfer either at 6 or $24 \mathrm{~h}$. These results suggest that treatment with Y-27632 at concentrations of $1 \mu \mathrm{M}$ or higher may be protective against endothelial damage induced by TNF- $\alpha$.

\section{Morphologic Changes in Endothelial Cell Monolayer}

Rhodamine-phalloidin staining of monolayers was performed to permit visualization of actin filaments. Control cells showed characteristic peripheral bands and close cell-cell contact (Figure $5 \mathrm{~A})$. Treatment with TNF- $\alpha$ at concentration of $10^{3} \mathrm{U} / \mathrm{ml}$ caused a significant change in this pattern. $\mathrm{TNF}-\alpha$ treatment resulted in the development of randomly oriented stress fibers, disappearance of peripheral bands, cell retraction, and intercellular gaps (Figure 5B). Pretreatment with Y-27632 $(100 \mu \mathrm{M})$ prevented the changes caused by exposure to TNF- $\alpha$ (Figure $5 \mathrm{C}$ ).

\section{DISCUSSION}

This study revealed that pretreatment with Y-27632 attenuated lung edema formation and neutrophil emigration after intravenous LPS. Although Y-27632 caused no change in fMLP-induced cytoskeletal rearrangement in neutrophils, morphologic changes in endothelial cells after TNF- $\alpha$ stimulation were inhibited by Y-27632 treatment. The TNF- $\alpha$-induced increase in albumin leakage through an endothelial monolayer was also attenuated by treatment with Y-27632. These results suggest a possible contribution of Rho/ROCK to the pathogenesis of LPS-induced acute lung injury.

LPS-induced lung injury is known to be neutrophil-dependent $(3,4)$. Intravenous or intratracheal challenge of inflammatory stimuli, such as LPS, has been shown to cause sequestration and subsequent emigration of neutrophils, which produce tissue injury by releasing various mediators and oxygen radicals (4). Rapid sequestration of neutrophils into the lung microvasculature after intravenous LPS administration is believed to depend on cell stiffening caused by the cytoskeletal rearrangement of neutrophils, whereas various adhesion molecules contribute to subsequent firm adhesion between neutrophils and endothelial cells (4). This study determined whether the effects of Y-27632 occurred through inhibition of neutrophil sequestration by preventing the formation of F-actin rims induced by mediators associated with gram-negative sepsis. Stimulating neutrophils with fMLP increases their F-actin content rapidly within the periphery of the neutrophils by $2 \mathrm{~min}$, resulting in neutrophil stiffening (26-28). In the present study, F-actin formation in human neutrophils was examined by staining with rhodamine-phalloidin, and an increase in the percentage in neutrophils containing F-actin rims was observed after fMLP stimulation. Although Y-27632 clearly modulates cytoskeletal rearrangements in smooth muscle cells and endothelial cells $(17,29)$, our observations revealed that Y-27632 did not alter the rapid submembrane formation of F-actin in nonadherent neutrophils stimulated with fMLP. Y-27632 also had no significant effect on the circulating neutrophil 

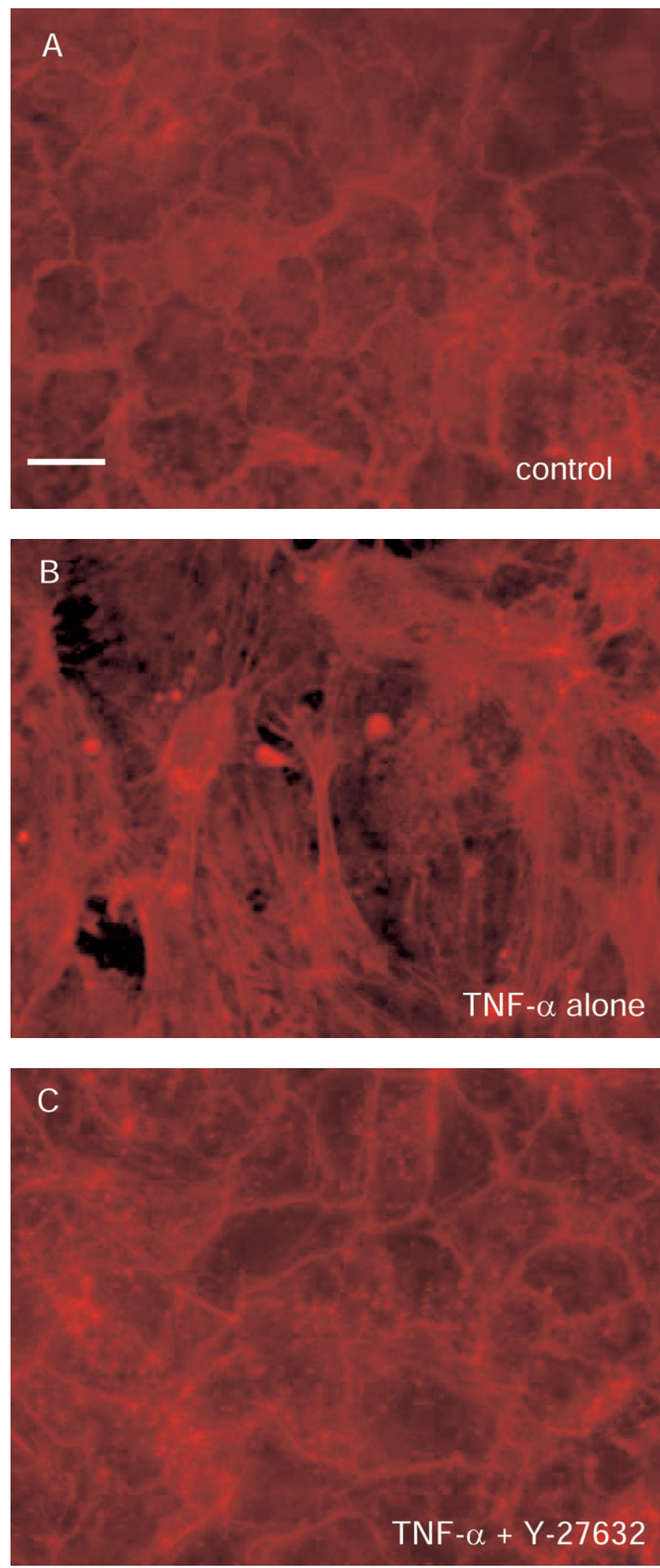

Figure 5. Rhodamine-phalloidin staining of endothelial cell monolayer. $(A)$ Control cells showing characteristic peripheral bands and close cell-cell contact. $(B)$ Cells treated with TNF- $\alpha\left(10^{3} \mathrm{U} / \mathrm{ml}\right)$ revealing development of randomly oriented stress fibers, disappearance of peripheral bands, cell retraction, and intercellular gaps. (C) Pretreatment with Y-27632 $(100 \mu \mathrm{M})$ prevented the changes induced by TNF- $\alpha$. Original bar, $20 \mu \mathrm{m}$. counts in uninjured mice and had no effect on the LPS-induced decrease in counts, suggesting that Y-27632 does not cause a significant increase in neutrophil stiffness or in neutrophil sequestration. Thus, the Rho/ROCK pathway does not appear to mediate neutrophil sequestration.

Following sequestration, however, many complex cytoskeletal events occur that mediate adhesion and crawling of neutrophils along endothelial cells and into the lung tissue. For example, activation of RhoA and ROCK is essential for de-adhesion or detachment of already adherent leukocytes $(30,31)$. Leukocytes pretreated with Y-27632 demonstrated impaired de-adhesion, which could be responsible for attenuated transmigration through the endothelium $(30,31)$. Although the effect of Y-27632 on leukocyte de-adhesion was not examined in this study, impairment of this process or others might be a mechanism for the attenuation of lung injury that we observed in animals treated with Y-27632.

The protective effect of Y-27632 on endothelial cells may be responsible for the attenuation of lung injury. To examine this hypothesis, the effect of Y-27632 on endothelial cell damage induced by $\mathrm{TNF}-\alpha$ was evaluated using cultured endothelial cell monolayers. TNF- $\alpha$ increased permeability through the monolayer, as estimated by albumin transfer, and induced actin remodeling visualized by rhodamine-phalloidin staining of the monolayer. Pretreatment with Y-27632 significantly inhibited these changes, suggesting that the Rho/ROCK pathway mediates these TNF- $\alpha$ effects.

The molecular motor that drives nonmuscle actin cytoskeletal rearrangement is the myosin II-associated ATPase, which generates mechanical force by promoting the translational crossbridge movement of myosin heads across the actin fibers. This process is regulated by the phosphorylation of myosin light chain (MLC) catalyzed by the $\mathrm{Ca}^{2+} /$ calmodulin-dependent MLC kinase (MLCK). MLC phosphorylation triggers myosin ATPase activity and actin polymerization and is essential to smooth muscle and nonmuscle tension development. Rho kinases increase MLC phosphorylation via inhibition of the regulatory subunit of the myosin-specific phosphatase, and inhibition of Rho-ROCK pathway decreases MLC phosphorylation (32). Petrache and colleagues reported that reduction in MLC phosphorylation by the inhibition of either MLCK or ROCK attenuated TNF$\alpha$-induced stress fiber formation (33). In their study, however, ROCK inhibition did not attenuate TNF- $\alpha$-induced endothelial barrier dysfunction measured by transcellular electrical resistance. In contrast, our studies demonstrated a complete inhibition of the TNF- $\alpha$-induced increase in permeability to albumin by the ROCK inhibitor. The most interesting and likely interpretation for these discrepant results in the role of ROCK in endothelial barrier function is that ROCK is required for disruption of permeability to protein, but decreases in electrical resistance can occur through ROCK-independent mechanisms. Other differences in the details of the studies may also contribute to the apparent discrepancy.

Because our in vitro study suggests that the inhibition of Rho/ROCK pathway protects against TNF-induced endothelial damage, the attenuation of the LPS-induced increase in EVA by Y-27632 observed in vivo may be due to an important role of this signaling pathway in endothelial cells of the pulmonary microvasculature. Saito and colleagues demonstrated that pretreatment with Y-27632 significantly attenuated leukotriene B4induced actin polarization in endothelial cells (29). Furthermore, although Y-27632 had no effect on the number of adherent neutrophils, neutrophil transmigration through endothelial cell monolayers was less when cells were pretreated with Y-27632 (29). Although we did not conduct a directly relevant experiment such as evaluation of neutrophil transmigration, the results of 
the present study, combined with previous findings, suggest that the Rho/ROCK pathway may be important in neutrophil migration or transendothelial cell migration, rather than neutrophil sequestration into the lung microvasculature. It remains to be determined whether Rho/ROCK inhibition modulates the function of neutrophils, endothelial cells, or both.

In the present study, we observed a significant decrease in the circulating neutrophil counts after LPS injection. Although in rats, rabbits, and guinea pigs, an intravenous challenge of LPS or other inflammatory mediators causes a rapid decline in circulating neutrophil count, there have been few reports describing neutrophil counts after inflammatory stimuli in mice (3, 34-36). Recently, Andonegui and colleagues reported a significant decrease in circulating leukocyte count $30 \mathrm{~min}$ after intraperitoneal administration of LPS in C57BL/6 mice (37). They also described LPS-induced neutrophil sequestration into the lungs, which was determined by MPO level in lung tissue (37). Although they did not present data describing circulating neutrophils, and our study did not quantitate neutrophil sequestration at early time points when the circulating neutrophil counts are low, this fall in circulating neutrophil counts likely reflects LPS-induced sequestration of neutrophils in the lungs.

As an inflammatory stimulus, we chose LPS for the murine model of acute lung injury, fMLP for neutrophil cytoskeleton observation, and TNF- $\alpha$ for endothelial monolayer studies. Because endotoxemia is known to be one of major predisposing factors of ARDS, LPS is widely used in animal models of experimental lung injury. In the present study, therefore, we used LPS to induce acute lung injury in mice so that we can compare the results with those from other studies. On the other hand, previous works suggest that the onset of neutrophil stiffening induced by fMLP and by LPS may differ. For example, whereas Saito and colleagues reported that neutrophil stiffness is rapidly (i.e., within $30 \mathrm{~s}$ ) increased after fMLP stimulation (34), Erzurum and colleagues revealed that LPS-induced neutrophil stiffness takes 10-30 min (38). Because the LPS-induced neutrophil stiffening takes a wide range of time, we thought it could be difficult to set a time point to estimate percentage of neutrophils with cytoskeletal rearrangement and, therefore, chose fMLP, which induces neutrophil stiffening readily within a minute, as a stimulus. Concerning the endothelial monolayer experiment, we performed a preliminary experiment using LPS as a stimulant (data not shown) and observed that LPS-induced changes in the permeability of endothelial monolayer were relatively irreproducible. In the endothelial monolayer experiments, therefore, we chose TNF- $\alpha$, which caused endothelial damage reproducibly, as a stimulant.

Pretreatment with Y-27632 significantly attenuated lung edema and neutrophil emigration in LPS-induced lung injury in mice, but it failed to reveal complete inhibition of lung injury. It was suggested that, even if this compound can be applicable as therapeutic modality for ARDS, its protective effect may be modest or limited. In addition, although we observed no symptom suggesting systemic hypotension in this study, it has been reported that Y-27632 inhibits smooth-muscle contraction and corrects the blood pressure of hypertensive rat models (17). When clinical application of Rho/ROCK inhibitor is considered, it should be necessary to preclude its possible detrimental effects (such as hypotension) and to assess the efficacy of Rho/ROCK pathway inhibition in established acute lung injury, not merely as a pretreatment.

In conclusion, Y-27632 attenuates LPS-induced acute lung injury, both the edema formation and the emigration of neutrophils. These effects are unlikely to be modulated through a role for the Rho/ROCK pathway in neutrophil sequestration. Rather, this compound may be acting through effects on modulating endothelial cell damage induced by inflammatory stimuli. Y-27632 may have potential as a therapeutic modality for acute lung injury.

Conflict of Interest Statement: S.T. has no declared conflicts of interest; H.K. has no declared conflicts of interest; W.Y. has no declared conflicts of interest; M.S. has no declared conflicts of interest; Y.O. has no declared conflicts of interest; N.H. has no declared conflicts of interest; K.Y. has no declared conflicts of interest; Y.I. has no declared conflicts of interest; S.E.R. has no declared conflicts of interest; C.M.D. has no declared conflicts of interest; A.I. has no declared conflicts of interest.

Acknowledgments: The authors thank Dr. Makoto Yonemaru of National Higashi Saitama Hospital for his expertise in cultured endothelial cell monolayers. They also thank Dr. Hajime Saito and Dr. Yoshitaka Minamiya of Akita University for much helpful discussion about the biomechanical properties of neutrophils. The authors thank Dr. Michio Terasawa of Mitsubishi Pharma Corporation for providing Y-27632.

\section{References}

1. Ashbaugh DG, Bigelow DB, Petty TL, Levine BE. Acute respiratory distress in adults. Lancet 1967;2:319-323.

2. Petty TL, Ashbaugh DG. The adult respiratory distress syndrome: clinical features, factors influencing prognosis and principles of management. Chest 1971;60:233-239.

3. Hogg JC, Doerschuk CM. Leukocyte traffic in the lung. Annu Rev Physiol 1995;57:97-114.

4. Doerschuk CM, Mizgerd JP, Kubo H, Qin L, Kumasaka T. Adhesion molecules and cellular biomechanical changes in acute lung injury. Chest 1999;116:37S-43S.

5. Saito H, Lai J, Rogers R, Doerschuk CM. Mechanical properties of rat bone marrow and circulating neutrophils and their responses to inflammatory mediators. Blood 2002;15:2207-2213.

6. Kubo H, Doyle NA, Graham L, Bhagwan SD, Quinlan WM, Doerschuk CM. L- and P-selectin and CD11/CD18 in intracapillary neutrophil sequestration in rabbit lungs. Am J Respir Crit Care Med 1999;159:267274.

7. Mizgerd JP, Kubo H, Kutkoski GJ, Bhagwan SD, Scharffetter-Kochanek $\mathrm{K}$, Beaudet AL, Doerschuk CM. Neutrophil emigration in the skin, lungs, and peritoneum: different requirements for CD11/CD18 revealed by CD18-deficient mice. J Exp Med 1997;186:1357-1364.

8. Motosugi H, Graham L, Noblitt TW, Doyle NA, Quinlan WM, Li Y, Doerschuk CM. Changes in neutrophil actin and shape during sequestration induced by complement fragments in rabbits. Am J Pathol 1996;149:963-973.

9. Mackay DJG, Hall A. Rho GTPases. J Biol Chem 1998;273:20685-20688.

10. Ridley AJ, Hall A. The small GTP-binding protein Rho regulates the assembly of focal adhesions and actin stress fibers in response to growth factors. Cell 1992;70:389-399.

11. Essler M, Amano M, Kruse H, Kaibuchi K, Weber PC, Aepfelbacher M. Thrombin inactivates myosin light chain phosphatase via Rho and its target Rho kinase in human endothelial cells. J Biol Chem 1998;273: 21867-21874.

12. Niggli V. Rho-kinase in human neutrophils: a role in signaling for myosin light chain phosphorylation and cell migration. FEBS Lett 1999;445: 69-72.

13. Kimura K, Ito M, Amano M, Chihara K, Fukata Y, Nakafuku M, Yamamori B, Feng J, Nakano T, Okawa K, et al. Regulation of myosin phosphatase by Rho and Rho-associate kinase (Rho kinase). Science 1996;273: $245-248$.

14. Maekawa M, Ishizaki T, Boku S, Watanabe N, Fujita A, Iwamatsu A, Obinata T, Ohashi K, Mizuno K, Narumiya S. Signaling from Rho to the actin cytoskeleton through protein kinases ROCK and LIM-kinase. Science 1999;285:895-898.

15. Narumiya S, Ishizaki T, Uehata M. Use and properties of ROCK-specific inhibitor Y-27632. Methods Enzymol 2000;325:273-284.

16. Ishizaki T, Uehata M, Tamechika I, Keel J, Nonomura K, Maekawa M, Narumiya S. Pharmacological properties of Y-27632, a specific inhibitor of rho-associated kinases. Mol Pharmacol 2000;57:976-983.

17. Uehata $M$, Ishizaki $T$, Satoh $H$, Ono $T$, Kawahara $T$, Morishita $T$, Tamakawa H, Yamagami K, Inui J, Maekawa M, et al. Calcium sensitization of smooth muscle mediated by a Rho-associated protein kinase in hypertension. Nature 1997;389:990-994.

18. Iizuka K, Shimizu Y, Tsukagoshi H, Yoshii A, Harada T, Dobashi K, Murozono T, Nakazawa T, Mori M. Evaluation of Y-27632, a Rhokinase inhibitor, as a bronchodilator in guinea pigs. Eur J Pharmacol 2000;406:273-279. 
19. Tasaka S, Richer SE, Mizgerd JP, Doerschuk CM. Very late antigen-4 in $\mathrm{CD} 18$-independent neutrophil emigration during acute bacterial pneumonia in mice. Am J Respir Crit Care Med 2002;166:53-60.

20. Kumasaka T, Doyle NA, Quinlan WM, Graham L, Doerschuk CM. Role of CD 11/CD 18 in neutrophil emigration during acute and recurrent Pseudomonas aeruginosa-induced pneumonia in rabbits. Am J Pathol 1996;148:1297-1305.

21. Goldblum SE, Wu KM, Jay M. Lung myeloperoxidase as a measure of pulmonary leukostasis in rabbits. J Appl Physiol 1985;59:1978-1985.

22. Soejima K, Fujishima S, Nakamura H, Waki Y, Nakamura M, Matsubara H, Tasaka S, Sayama K, Ishizaka A, Kanazawa M. Downmodulation of IL-8 receptors, type A and type B, on human lung neutrophils in vivo. Am J Physiol 1997;273:L618-L625.

23. Reibman J, Meixler S, Lee TC, Gold LI, Cronstein BN, Haines KA Kolasinski SL, Weissmann G. Transforming growth factor beta 1, a potent chemoattractant for human neutrophils, bypasses classic signaltransduction pathways. Proc Natl Acad Sci USA 1991;88:6805-6809.

24. Yonemaru M, Kasuga I, Kusumoto H, Kiyokawa H, Kuwabara S, Ichinose Y, Toyama K. Protein kinase inhibitor attenuates an increase in endothelial monolayer permeability induced by tumour necrosis factor-alpha. Respirology 1997;2:63-69.

25. Phillips PG, Tsan MF. Direct staining and visualization of endothelial monolayers cultured on synthetic polycarbonate filters. J Histochem Cytochem 1988;36:551-554.

26. Howard TH, Oresajo CO. The kinetics of chemotactic peptide-induced change in F-actin content, F-actin distribution, and the shape of neutrophils. J Cell Biol 1985;101:1078-1085.

27. Erzurum SC, Kus ML, Bohse C, Elson EL, Worthen GS. Mechanical properties of HL60 cells: role of stimulation and differentiation in retention in capillary-sized pores. Am J Respir Cell Mol Biol 1991;5: 230-241.

28. Wang Q, Chiang ET, Lim M, Lai J, Rogers R, Janmey PA, Shepro D, Doerschuk CM. Changes in the biomechanical properties of neutrophils and endothelial cells during adhesion. Blood 2001;97:660-668.
29. Saito H, Minamiya Y, Saito S, Ogawa J. Endothelial Rho and Rho kinase regulate neutrophil migration via endothelial myosin light chain phosphorylation. J Leukoc Biol 2002;72:829-836.

30. Alblas J, Ulfman L, Hordijk P, Koenderman L. Activation of RhoA and ROCK are essential for detachment of migrating leukocytes. Mol Biol Cell 2001;12:2137-2145.

31. Liu L, Schwartz BR, Lin N, Winn RK, Harlan JM. Requirement for RhoA kinase activation in leukocyte de-adhesion. J Immunol 2002; 169:2330-2336.

32. Garcia JG, Verin AD, Schaphorst K, Siddiqui R, Patterson CE, Csortos C, Natarajan V. Regulation of endothelial cell myosin light chain kinase by Rho, cortactin, and $\mathrm{p}^{\circ} 0^{\mathrm{sc}}$. Am J Physiol Lung Cell Mol Physiol 1999;276:L989-L998.

33. Petrache I, Verin AD, Crow MT, Birukova A, Liu F, Garcia JG. Differential effect of MLC kinase in TNF-alpha-induced endothelial cell apoptosis and barrier dysfunction. Am J Physiol Lung Cell Mol Physiol 2001;280:L1168-L1178.

34. Saito H, Lai J, Rogers R, Doerschuk CM. Mechanical properties of rat bone marrow and circulating neutrophils and their responses to inflammatory mediators. Blood 2002;99:2207-2213.

35. Klut ME, van Eeden SF, Whalen BA, Verburgt LM, English D, Hogg JC. Neutrophil activation and lung injury associated with chronic endotoxemia in rabbits. Exp Lung Res 1996;22:449-465.

36. Tasaka S, Ishizaka A, Urano T, Sayama K, Sakamaki F, Nakamura H, Terashima T, Waki Y, Soejima K, Oyamada Y, et al. BCG priming enhances endotoxin-induced acute lung injury independent of neutrophils. Am J Respir Crit Care Med 1995;152:1041-1049.

37. Andonegui G, Bonder CS, Green F, Mullaly SC, Zbytnuik L, Raharjo E, Kubes P. Endothelium-derived Toll-like receptor-4 is the key molecule in LPS-induced neutrophil sequestration into lungs. J Clin Invest 2003;111:1011-1020.

38. Erzurum SC, Downey GP, Doherty DE, Schwab B, Elson EL, Worthen GS. Mechanisms of lipopolysaccharide-induced neutrophil retention: relative contributions of adhesive and cellular mechanical properties. J Immunol 1992;149:154-162. 